\title{
Article
}

\section{Time recovery for a complex process using accelerated dynamics}

\author{
Sergio Alexis Paz, and Ezequiel Pedro Marcos Leiva
}

J. Chem. Theory Comput., Just Accepted Manuscript • DOI: 10.1021/ct5009729 • Publication Date (Web): 24 Feb 2015

Downloaded from http://pubs.acs.org on March 12, 2015

\section{Just Accepted}

"Just Accepted" manuscripts have been peer-reviewed and accepted for publication. They are posted online prior to technical editing, formatting for publication and author proofing. The American Chemical Society provides "Just Accepted" as a free service to the research community to expedite the dissemination of scientific material as soon as possible after acceptance. "Just Accepted" manuscripts appear in full in PDF format accompanied by an HTML abstract. "Just Accepted" manuscripts have been fully peer reviewed, but should not be considered the official version of record. They are accessible to all readers and citable by the Digital Object Identifier (DOI®). "Just Accepted" is an optional service offered to authors. Therefore, the "Just Accepted" Web site may not include all articles that will be published in the journal. After a manuscript is technically edited and formatted, it will be removed from the "Just Accepted" Web site and published as an ASAP article. Note that technical editing may introduce minor changes to the manuscript text and/or graphics which could affect content, and all legal disclaimers and ethical guidelines that apply to the journal pertain. ACS cannot be held responsible for errors or consequences arising from the use of information contained in these "Just Accepted" manuscripts. 


\title{
Time recovery for a complex process using
}

\section{accelerated dynamics}

\author{
S. Alexis Paz ${ }^{*,+\ddagger}$ and Ezequiel P. M. Leiva ${ }^{\dagger}$ \\ INFIQC - Departamento de Matemática y Fúsica, Facultad de Ciencias Químicas, \\ Universidad Nacional de Córdoba, Córdoba, Argentina., and Department of Chemical and \\ Biological Engineering, Drexel University, Philadelphia, PA 19104, United States. \\ E-mail: apaz@fcq.unc.edu.ar
}

\begin{abstract}
The hyperdynamics method (HD) developed by Voter [J. Chem. Phys. 106 (1996) 11] sets the theoretical basis to construct an accelerated simulation scheme that holds the time scale information. Since HD is based on transition state theory, pseudoequilibrium condition (PEC) must be satisfied before any system in a trapped state may be accelerated. As the system evolves, many trapped states may appear and the PEC must be assumed in each one to accelerate the escape. However, since the system evolution is a priori unknown, the PEC cannot be permanently assumed as true. Furthermore, the different parameters of the bias function used may need drastic recalibration during this evolution. To overcome this problems we present a general scheme to switch between HD and conventional molecular dynamics (MD) in an automatic fashion during the simulation. To decide when HD should start and finish, criteria

\footnotetext{
${ }^{*}$ To whom correspondence should be addressed

${ }^{\dagger}$ INFIQC - Departamento de Matemática y Física, Facultad de Ciencias Químicas, Universidad Nacional de Córdoba, Córdoba, Argentina.

${ }^{\ddagger}$ Department of Chemical and Biological Engineering, Drexel University, Philadelphia, PA 19104, United States. 
based on the energetic properties of the system are introduced. On the other hand, a very simple bias function is proposed leading to a straightforward on-the-fly set up of the required parameters. A way to measure the quality of the simulation is proposed. The efficiency of the present hybrid HD-MD method is tested for a two-dimensional model potential and for the coalescence process of two nanoparticles. In spite of the important complexity of the latter system (165 degrees of freedoms), some relevant mechanistic properties were recovered within the present method.

\section{Introduction}

One of the biggest problems of computer simulations using molecular dynamics (MD) is the relatively short time scale that can be reached within this method. To achieve long simulation times, powerful computer clusters are needed, and even in this case, only a few microseconds can be simulated. For many systems, the dynamics at long times is characterized by rare (infrequent) events: the system must escape from a certain basin of its potential energy surface (PES) after wasting a long time trapped in it. At the present, many tools to handle these rare events have been developed. For instance, there are different methods that allow an enhanced sampling of the free energy surface (FES) associated with one or more specific collective variables $(\mathrm{CVs})$ of the system. ${ }^{1-10}$ In general, suitable CVs are chosen from a prior knowledge of the system. On the other hand, the hyperdynamics (HD) developed by $\operatorname{Voter}^{11,12}$ aims to achieve an acceleration in a representative way of the exact dynamics of the system. In this case, the time information and the power of prediction of the conventional MD can be retained.

Following the importance sampling, ${ }^{13}$ the HD introduces a transformation of the PES by the addition of a bias function $\Delta U_{b}$ :

$$
U_{b}(\mathbf{r})=U(\mathbf{r})+\Delta U_{b}(\mathbf{r})
$$


While this transformation leads to a reduction of the energy barriers of the PES, the residence time in the metastable states will be shorter, improving the occurrence frequency of the rare events. Using transition state theory (TST), Voter showed that the correct long-time kinetics of the system can be recovered if the bias function is designed to vanish at the transition states (TS). In spite of the great potentiality of this method, its implementation is not straightforward, as can be seen from the many different approaches made in the last fifteen years. ${ }^{14-30}$ For instance, finding a bias function that vanishes at the TS is a non-trivial task, especially for systems with many degrees of freedom.

Since HD is based on TST, the system is required to achieve the pseudo-equilibrium condition (PEC) at the trapped state before the HD may be applied. If the system is well known, to ensure this condition for any state (as it is the case of a Markovian process) is not a major problem. However, in the opposite case, an on-the-fly algorithm should be applied to determine when HD can be safely used. In addition, the use of a bias function usually requires different parameters that must be fixed for the particular state of the system. These parameters might be unknown or even need drastic recalibration as the evolution of the system takes place. Therefore, an acceleration algorithm with an automatic regulation of these parameters is needed. For instance, Perez and Voter ${ }^{19}$ used a self-learning algorithm to safely parametrize a variation of the bond boost method of Miron and Fichthorn. ${ }^{25}$

In the present work we propose an hybrid HD-MD scheme to parametrize on-the-fly energy-based bias functions similar to that proposed by Hamelberg et al. ${ }^{23}$ During a MD simulation period a test for the PEC is continually performed. When this test is successful, the parameters of the bias function are automatically fixed using local energy properties of the trapped state and some generals rules for the wished acceleration. Then, the HD simulation starts and continues until a maximum limit for the magnitude of the bias (i.e. the instantaneous value of the bias function) is overcome. In general this limit leads to a decay probability for the HD simulation, but it also acts as a fuse if the acceleration becomes too aggressive indicating that a recalibration of the bias parameters is needed. Finally, the 
MD simulation starts again and the described process is iterated.

This work is organized as follows. The method to analyze the occurrence of PEC is presented in section 2. In section 3 we present the simple bias function used for the present implementation and in section 4 we indicate how the parameters required are adjusted onthe-fly. The criterion to finish the HD simulation is presented in section 5 and a global scheme of the hybrid HD-MD algorithm is given in section 6. Finally, we illustrate the use of the method for two different systems. First, we use the method to recover the drift and diffusion coefficient in a simple two-dimensional potential surface. Second, we apply it to study the coalescence process of metal nanoparticles (NPs). The results are compared directly with those obtained in our recent work ${ }^{31}$ by long MD simulations. The application of the present HD scheme allow us to recover useful time information for a complex process with a relatively large number of degrees of freedom (165). In spite of the good performance of the present method, further improvements are finally discussed and the need for a deeper study on the scope and limitations of the hybrid HD-MD method is stated.

\section{Analysis of the attainment of pseudo-equilibrium con- ditions}

Since HD is based on TST, it is necessary that the system achieves pseudo-equilibrium conditions (PEC) at the trapped state before HD is applied. Here we propose a criterion to define when PEC are reached based on the statistical behavior of the potential energy.

Let $X$ be the potential energy of a system monitored during a canonical MD simulation. The value of $X$ at the $i$ th step of the simulation will be denoted as $X_{i}$. For a set of MD simulations, each one with $N$ steps, the average value $\bar{X}=\sum_{i}^{N} X_{i} / N$ has a variance given 


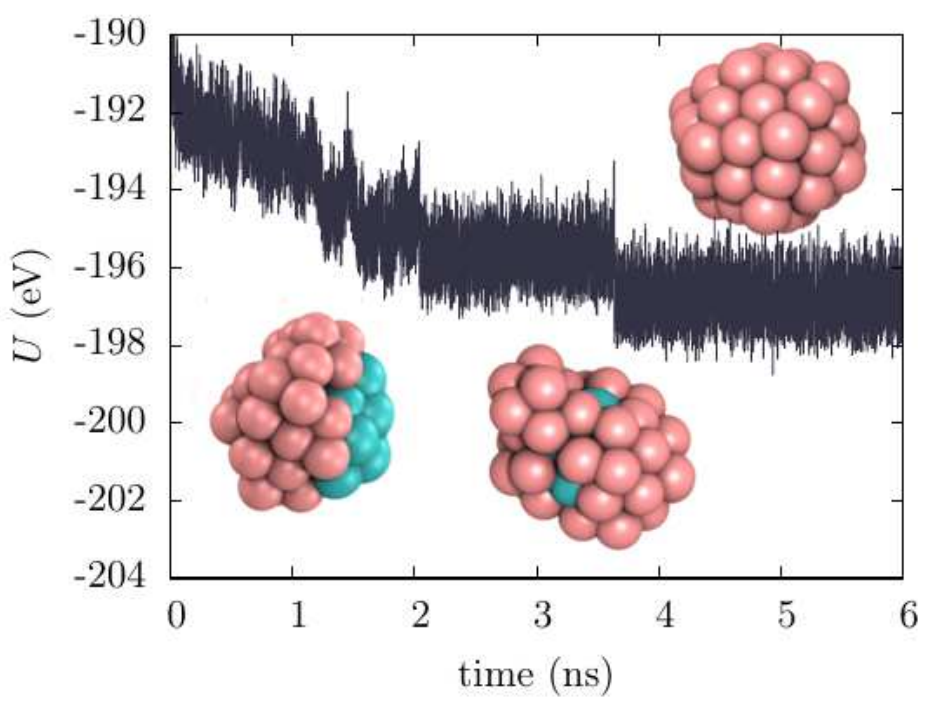

Figure 1: Energy trace for the coalescence process of two metallic NPs, made of $42 \mathrm{Au}$ atoms and 13 Co atoms respectively. Au atoms are shown in pink and Co atoms in blue.

by:

$$
\sigma^{2}(\bar{X})=\frac{\sigma^{2}(X)}{N}+\frac{2}{N^{2}} \sum_{j>i}^{N} \sigma\left(X_{i}, X_{j}\right)
$$

where $\sigma\left(X_{i}, X_{j}\right)$ is the covariance for the pairs $X_{i}$ and $X_{j}$ along the different MD simulations. To avoid the computation of the covariance term, a decorrelation process on the data trace from each MD simulation is commonly used. This process involves splitting each trace in $m$ blocks and using the averages over each block as a set of $\mathcal{X}_{i}, \ldots, \mathcal{X}_{m}$ decorrelated energy values per MD simulation. If $N$ is sufficiently large, the minimum value of $m$ required to get a decorrelated data set can be obtained from a Flyvbjerg-Petersen Plot (FPP), ${ }^{32}$ which shows the dependence with $m$ of

$$
\sigma_{m}=\sqrt{\frac{\sigma(\mathcal{X})}{m-1}}\left(1 \pm \frac{1}{\sqrt{2(m-1)}}\right)
$$

This quantity increases with $m$ until a certain value is reached and then it remains constant within fluctuations. This plateau is an estimator for $\sigma(\bar{X})$ and its presence indicates that a decorrelated data set was obtained by the blocking operation. From another point of view, 
the plateau in the FPP serves as an indicator that the system has a well behaved energy distribution with meaningful values for $\bar{X}$ and $\sigma(X)$. A well behaved energy distribution is a characteristic of systems that have reached pseudo-equilibrium conditions (PEC). Therefore we propose to use the occurrence of the plateau in the FPP as prerequisite to start the HD simulation.

In Figure 1 we show the energy trace of a MD simulation obtained in a previous work. ${ }^{31}$ The process under study was the coalescence of two particles made of $42 \mathrm{Au}$ atoms and 13 Co atoms respectively. The trajectory arose from a Langevin dynamics at $550 \mathrm{~K}$ (friction coefficient of $5 \mathrm{ps}^{-1}$ ) using second moment approximation to the tight binding potentials (parameters from ${ }^{33}$ ). It is noted in this figure the initial decrease of the energy due to the formation of the new Au-Co bonds and the steady state reached after $\approx 4$ ns due to the occurrence of the final core-shell structure. For the time intervals (1-2) and (4-5) ns we computed the respective FPPs, as shown in figure 2. The presence of a plateau is evident in figure $2 \mathrm{~b}$, in contrast with the exponential shape observed in figure $2 \mathrm{a}$.

The use of the criterion presented above to start the HD simulation has some advantages. First, the FPP can be constructed on-the-fly using the dynamic decorrelation distributable algorithm (DDDA) designed by Kent et al. ${ }^{34}$ This is a very efficient algorithm and can be even used in parallel computing schemes. Second, the test exhibits a relatively good sensitivity to small perturbations of the energy. Third, after the test is successfully evaluated, the $\bar{X}$ and $\sigma(X)$ of the particular trapped state are immediately available for use. For instance, these values can be used to setup the bias function parameters required, as described in section 4. Fourth, if the trapped state involves several local minima and small barriers, the criterion will still be useful if the energy trace involved is taken over a sufficiently large run. Beyond these advantages, it is important to emphasize that the proposed test is only a criterion and not a definitive test for the PEC. Although it is true that a plateau will be found in the FPP when the system reaches the PEC, the opposite might not be true as it happens with some pathological cases, like a system with a consistently flat PES. 


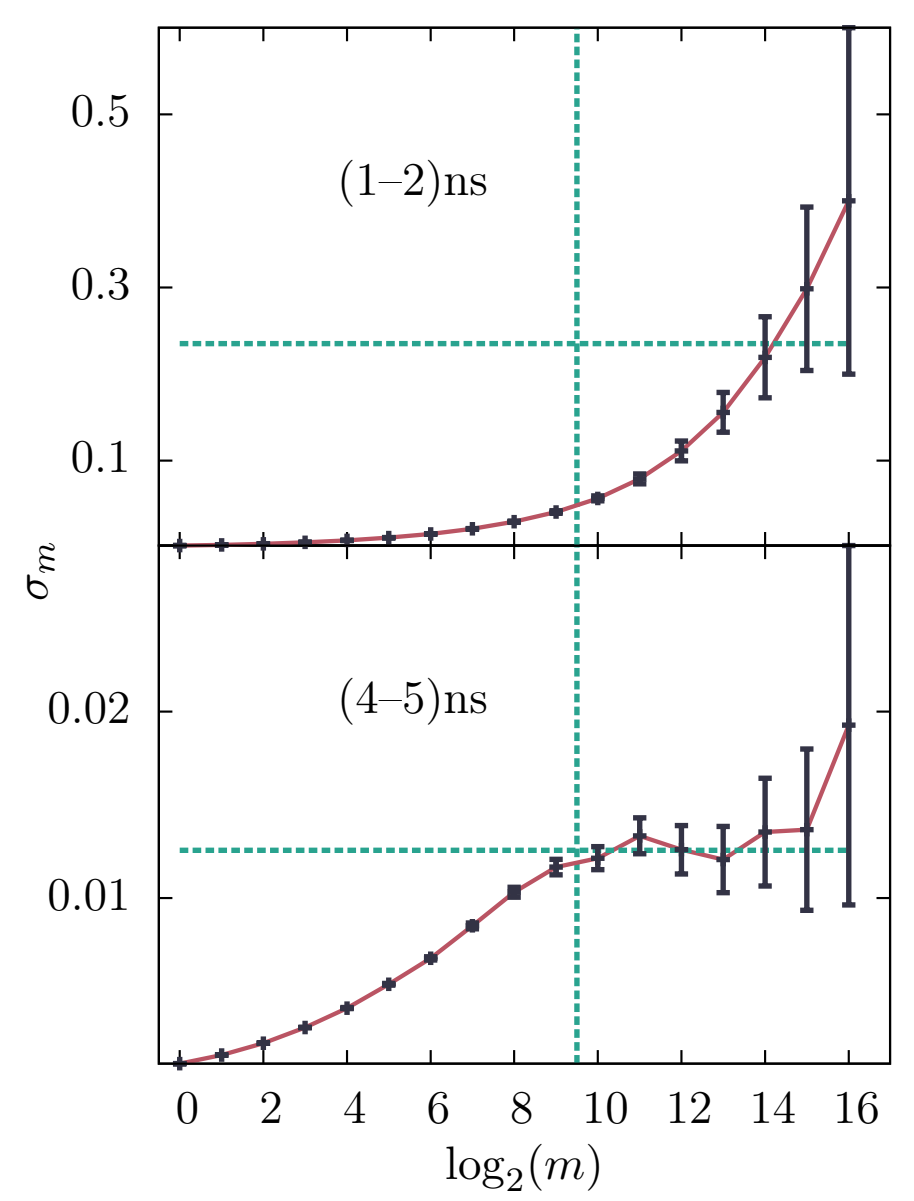

Figure 2: a): Flyvbjerg-Petersen plots (FPP) for the interval (1-2) ns of the energy trace shown in figure 1b): Same as a) but for the interval (4-5) ns. Vertical and horizontal lines are drawn to guide the eyes. 
After the FPP is obtained on-the-fly using the DDDA, an automatic way to detect the plateau in the plot is needed. In this work we use a simple procedure but other more sophisticated might be chosen. First, we measure the length of the candidate plateau as the maximum number of consecutive $m$ values, counted from the right side of the plot, for which the intersection of the error bars is not void. For example, the plateau in figure 2 a has only 3 consecutive $m$ values of length $\left(\log _{2}(m)=16,15,14\right)$ but in figure $2 \mathrm{~b}$ it extends over $7 \mathrm{~m}$ values $\left(\log _{2}(m)=16, \ldots, 10\right)$. If this length covers a certain (arbitrarily defined) fraction of the total $m$ values we consider that the FPP has a well defined plateau. The vertical lines in figure 2 indicates the defined fraction used in this work $(7 / 17)$.

\section{Selecting the bias function}

In order to start a HD simulation, a suitable bias function must be selected. Since we are using an energy-based test to verify the attainment of the PEC, an energy-based bias function is an appropriate choice. This kind of bias function satisfies:

$$
\Delta U_{b}(\mathbf{r})=g(U(\mathbf{r}))
$$

where $g$ is any arbitrary function. In order to keep a transparent notation we will write $g(x)$ also as $\Delta U_{b}(x)$, where the distinction with $\Delta U_{b}(\mathbf{r})$ will be evident from the nature of the argument. An useful energy-based bias function was proposed by Hamelberg et al.: ${ }^{23}$

$$
\Delta U_{b}(U)=\left\{\begin{array}{cc}
\frac{(E-U)^{2}}{\alpha+E-U} & U<E \\
0 & U \geq E
\end{array}\right.
$$

Where $E$ is a threshold energy above which the PES remains unchanged and $\alpha$ is a positive number that regulates the intensity of the boost. The parameter $E$ has an important meaning: any escape from the basin enclosed by the surface $U(\mathbf{r})=E$ satisfies Voter's condition 
for zero bias at the TS. Therefore, $E$ may be tunned to observe rare events in the desired time scale, while the other events inside the basin are assumed to be ergodically sampled as required by the PEC. However, when the number of degrees of freedom of the system (say $n$ ) is relatively large, the set up of $E$ is not trivial. The average energy and energy fluctuations grow with $n$, thus forcing to increase $E$ to get a reasonable acceleration. For these cases, Hamelberg and collaborators suggest to set up $E$ relative to the average energy. ${ }^{23}$ It must be emphasized that within the methodology presented above the statistical properties of the energy (as $\sigma(X)$ and $\bar{X}$ ) are immediately available after the FPP plateau is found.

For the sake of simplicity, instead of the Hamelberg et al. bias function we will use a simpler version given by:

$$
\Delta U_{b}(U)=\left\{\begin{array}{cc}
(E-U)(1-\alpha) & U<E \\
0 & U \geq E
\end{array}\right.
$$

with $0<\alpha \leq 1$. This function leads to the transformation:

$$
U_{b}(\mathbf{r})=\left\{\begin{array}{cc}
\alpha U(\mathbf{r})+E(1-\alpha) & U(\mathbf{r})<E \\
U(\mathbf{r}) & U(\mathbf{r}) \geq E
\end{array}\right.
$$

The linear relation between the biased and unbiased PES facilitates the analytical treatment of the HD scheme, as we will show in the next section. For instance, the parameter $\alpha$ has now a straightforward meaning, since it is the compression factor of any energy difference (i.e. barrier length) below $E$ (see figure 3). The parameter $E$ keeps the same meaning as before. The main drawback of this function with respect to that of Hamelberg et al. is the introduction of a discontinuity in the force when $U(\mathbf{r})=E$. However, since a stochastic dynamic is used to performed the HD simulation, we believe that this discontinuity does not introduce a major error in the results. We have checked that for some simple systems the present bias function delivers results similar to those obtained with the bias of equation 5 . It 
is important to remark that in any case the hybrid HD-MD algorithm here presented could be improved using the Hamelberg et al. bias function. The choice of the lineal transformation scheme was made to clarify the presentation of the method.

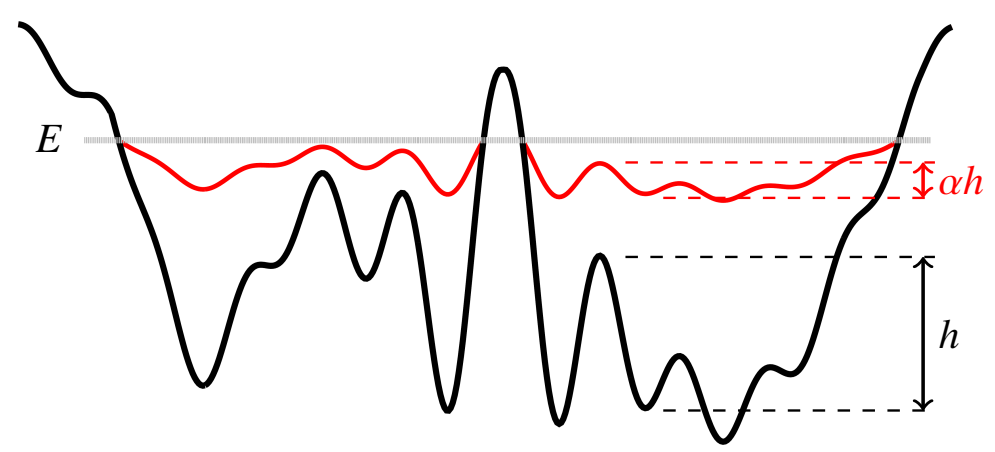

Figure 3: Example of the PES transformation obtained using the bias function defined in equation 6 .

\section{Setting the bias function parameters}

Prior to start with the HD simulation stage, a suitable choice of the parameters $E$ and $\alpha$ must be made. Since the energetic properties of the trapped states are a priori unknown, these parameters can not be kept fixed during the entire hybrid HD-MD simulation. For instance, a certain value of $E$ may be appropriate for a particular trapped state, but could be inappropriate for others. Therefore, an automatic way to set up $\alpha$ and $E$ when each HD simulation period begins should be derived from the general properties of the system and the acceleration desired.

In section 2 we defined $X$ as the potential energy of the system observed during a canonical MD simulation. $X$ can be envisaged as a random variable distributed according to:

$$
P(x)=\frac{e^{-\beta x} \omega(x)}{Q}
$$

where $\beta=k_{B} T, Q$ is the canonical partition function and $\omega(x)$ is the density of states. Similarly, we define $Y$ as the potential energy of the (biased) system observed during a HD 
simulation. The distribution function associated with $Y$ is:

$$
P_{b}(y)=\frac{e^{-\beta y} \omega_{b}(y)}{Q_{b}}
$$

where $\omega(y)_{b}$ and $Q_{b}$ are the density of states and the partition function of the biased system respectively. In the appendix we show a derivation of an expression relating $P_{b}(y)$ and $P(x)$ for any energy-based bias function. For the particular case of the selected bias function we get the relation:

$$
P_{b}(y)=\frac{Q}{Q_{b}}\left\{\begin{array}{cc}
\frac{1}{\alpha} e^{-\frac{\beta}{\alpha} \Delta U_{b}(y)} P\left(y-\frac{1}{\alpha} \Delta U_{b}(y)\right) & y<E \\
P(y) & y \geq E
\end{array}\right.
$$

Expression 10 allows us to set up on-the-fly the parameters $E$ and $\alpha$ of the bias function from the statistical properties of the previous MD simulation and the desired boost factor. In fact, the boost factor is given by:

$$
\begin{aligned}
B & \equiv \frac{Q}{Q_{b}}=\left\langle e^{\beta \Delta U_{b}(\vec{r})}\right\rangle_{b}=\left\langle e^{-\beta \Delta U_{b}(\vec{r})}\right\rangle^{-1} \\
& =\left[\int_{-\infty}^{\infty} e^{-\beta \Delta U_{b}(x)} P(x) d x\right]^{-1}
\end{aligned}
$$

Where \langle\rangle and \langle\rangle$_{b}$ are the averages over the canonical ensemble of the unbiased and biased systems respectively. The parameter $B$ does not depend on the particular trapped state of the system and is fixed from the acceleration requirements. Another parameter relevant for our purposes is:

$$
w \equiv \int_{-\infty}^{E} P_{b}(y) d y
$$

which gives the probability of observing a non-zero bias. This is a property of interest for the bias function presented in section 3. For instance, if $w$ is close to one Voter's condition 
concerning null bias at the TS will be compromised. On the other hand, if $w$ is too low (close to cero) a poor sampling of the biased region of the trapped state will take place. Hence, a value of $w$ near 0.5 could be desirable (see reference 11 ).

Replacing equation 10 in equation 12 yields:

$$
w=\frac{B}{\alpha} \int_{-\infty}^{E} e^{-\frac{\beta}{\alpha} \Delta U_{b}(x)} P\left(x-\frac{1}{\alpha} \Delta U_{b}(x)\right) d x
$$

Equations 11 and 13 constitute a system that enables to find $B$ and $w$ for given $E, \alpha$ and $P(x)$. Conversely, we will show that the parameters $E$ and $\alpha$ can be computed using these equations, once the global parameters $B$ and $w$ are fixed at the beginning of the hybrid HD-MD simulation.

Before starting the HD simulation period, the statistical properties of the energy, like $\bar{X}$ and $\sigma(X)$ may be obtained from the previous MD simulation. For energy values close to the maximum of $P(x)$, we can approximate this distribution by a normal distribution: ${ }^{35}$

$$
P(x) \approx \frac{1}{\sigma(X) \sqrt{2 \pi}} e^{-\frac{1}{2}\left(\frac{x-\bar{X}}{\sigma(X)}\right)^{2}}
$$

With this approximation equations 11 and 13 become:

$$
\left\{\begin{aligned}
B & =\left[1-\operatorname{cdf}_{\mathrm{sn}}\left(E^{\prime}\right)+\operatorname{cdf}_{\mathrm{sn}}\left(E^{\prime}-\alpha^{\prime}\right) \exp \left(\frac{\alpha^{\prime 2}}{2}-\alpha^{\prime} E^{\prime}\right)\right]^{-1} \\
w & =1-B\left(1-\operatorname{cdf}_{\mathrm{sn}}\left(E^{\prime}\right)\right)
\end{aligned}\right.
$$

where $\mathrm{cdf}_{\mathrm{sn}}$ is the cumulative distribution function for the standard normal distribution and

$$
\begin{aligned}
E^{\prime} & \equiv \frac{E-\bar{X}}{\sigma(X)} \\
\alpha^{\prime} & \equiv \beta \sigma(X)(1-\alpha) \quad 0<\alpha^{\prime}<\beta \sigma(X)
\end{aligned}
$$

Despite these new parameters were defined in terms of $E$ and $\alpha$, equation 15 indicates that 
these are directly related with $B$ and $w$. Therefore, $E^{\prime}$ and $\alpha^{\prime}$ are reduced versions of $E$ and $\alpha$ that can be taken as global parameters of the acceleration (i.e. can be fixed using equation 15 regardless the particular trapped state of the system). The maximum value $\alpha^{\prime}=\beta \sigma$ corresponds to $\alpha=0$ and can be estimated as $\sqrt{n / 2}$, where $n$ is the number of degrees of freedom of the system (this will be exactly true only for $n$ independent harmonic oscillators). Note that although this upper limit depends on $n$, equation 15 is valid for any system and temperature, allowing us to analyze the dependence of $B$ and $w$ on $E^{\prime}$ and $\alpha^{\prime}$ in a general way. We come now to this point.

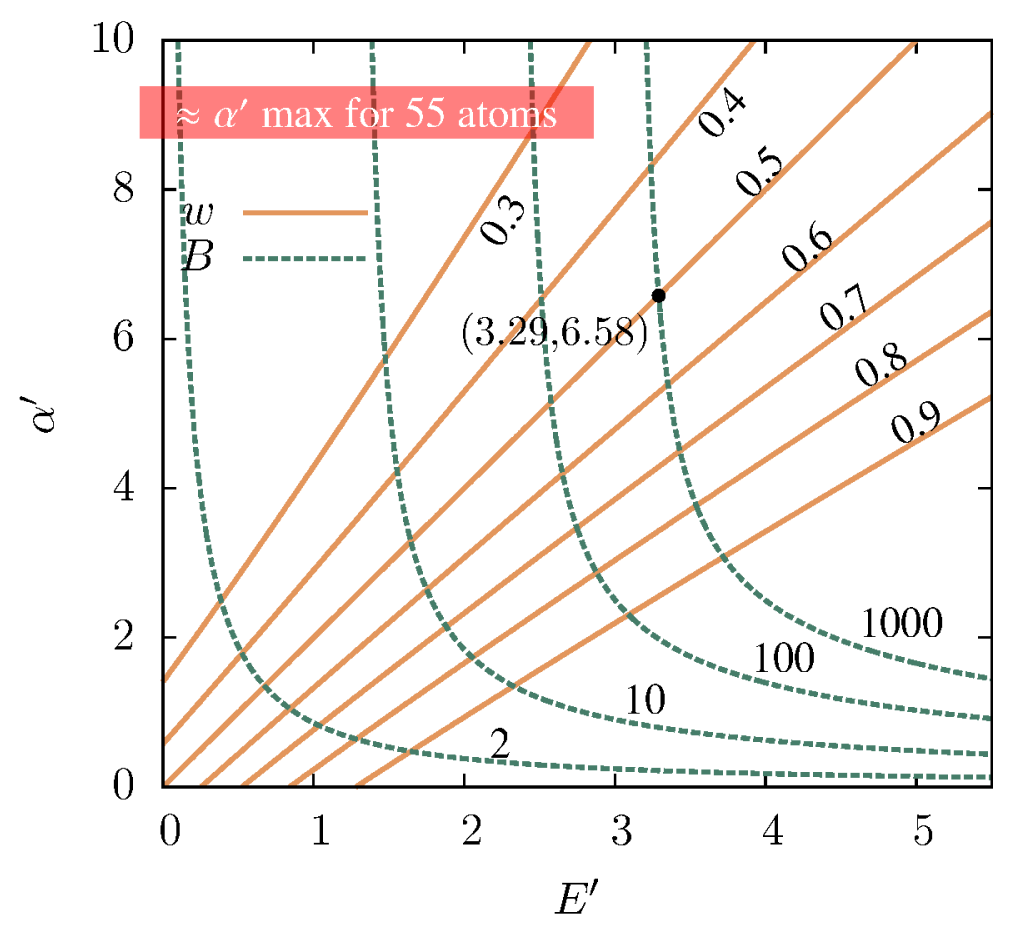

Figure 4: Contour map of $B$ and $w$ in function of $E^{\prime}$ and $\alpha^{\prime}$. The maximum boundary of $\alpha^{\prime}$ indicated is approximated from a system of 165 independent harmonic oscillators.

Figure 4 shows a contour map of $B$ and $w$ as a function of $E^{\prime}$ and $\alpha^{\prime}$. The point of intersection of the curves for given $B$ and $w$ correspond to the values of $E^{\prime}$ and $\alpha^{\prime}$ that should be chosen to yield the wished $B$ and $w$. For example, it can be found from this plot that if a boost factor of 1000 is required, with a probability of observing a non-zero bias of 0.5 , the values of $E^{\prime}=3.29$ and $\alpha^{\prime}=6.58$ must be chosen (see the point marked in figure 4). Thus, at any time that a HD simulation period is to be started, the $E$ and $\alpha$ must be 
obtained by inverting equations 16 and 17 . Important insights can be obtained from the plots in figure 4. For instance, when the system is big (large $\alpha^{\prime}$ values), the boost factor becomes insensitive to $\alpha^{\prime}$ and it is necessary to increase the value of $E^{\prime}$ in order to get a higher acceleration.

A final advantage can be pointed out for the present simulation scheme. The wished values for $B$ and $w$ are now input parameters for the hybrid HD-MD simulation. Furthermore, since $B$ and $w$ can also be directly measured during the simulation, the comparison between the target and the measured values may be taken as a degree of the quality of the simulation.

\section{Switching back to the MD from the HD}

In a similar way as we can predict the biased energy distribution $P_{b}(y)$, we can predict the probability distribution of the bias values observed, say $P_{\Delta}(z)$. That is, $P_{\Delta}(z) d z$ is the probability of observing a bias of a magnitude between $z$ and $z+d z$ in the course of the simulation. In the appendix we derive an expression relating $P_{\Delta}(z)$ with $P_{b}(y)$ for the bias function used in this work:

$$
P_{\Delta}(z)=\left\{\begin{array}{cc}
\frac{B}{1-\alpha} P\left(E-\frac{z}{1-\alpha}\right) e^{-\beta z} & z>0 \\
(1-w) \delta(z) & z=0
\end{array}\right.
$$

Therefore, the probability to observe a bias of magnitude $Z$ above a certain value $Z_{M}$ is:

$$
\begin{aligned}
\phi\left(Z_{M}\right) & =1-\int_{0}^{Z_{M}} P_{\Delta}(z) d z \\
& =B \operatorname{cdf}_{\mathrm{sn}}\left(E^{\prime}-\alpha^{\prime}-\frac{\beta}{\alpha^{\prime}} Z_{M}\right) \exp \left(\frac{\alpha^{\prime 2}}{2}-\alpha^{\prime} E^{\prime}\right)
\end{aligned}
$$

We will fix $Z_{M}$ as an initial parameter of the hybrid HD-MD algorithm in order to build a criterion to stop the HD simulation: when the magnitude of the bias goes over $Z_{M}$ the HD simulation switches to the ordinary MD. In other words, $Z_{M}$ will be a threshold limit for the 
bias magnitude, providing a fuse device to stop the HD simulation. $\phi=\phi\left(Z_{M}\right)$ becomes a decay probability for the HD simulation and the value of $Z_{M}$ can be fixed choosing $\phi$ and inverting the above equation:

$$
Z_{M}=\frac{\alpha^{\prime}}{\beta}\left[E^{\prime}-\alpha^{\prime}-\operatorname{cdf}_{\mathrm{sn}}^{-1}\left(\frac{\phi}{B} \exp \left(-\frac{\alpha^{2}}{2}+\alpha^{\prime} E^{\prime}\right)\right)\right]
$$

If $\phi$ has a relatively low value, the proposed criterion has the advantage to avoid the small barrier problem. ${ }^{24}$ That is, if the system escapes to a neighboring trapping state with similar energy, the HD simulation will continue without the need to recalibrate the parameters. However, if the new trapping state has a lower energy, the probability $\phi\left(Z>Z_{M}\right)$ will increase quickly and the HD will be terminated, allowing to start the MD and recalibrate the bias parameters for the new state.

\section{The global picture of the hybrid HD-MD method}

Figure 5 shows a complete diagram of the method proposed. Prior to starting the simulation it is necessary to define the time scale of interest, which leads to the set-up of the boost factor $B$. Also a value for the parameter $w$ must be chosen. We suggest for the latter a value close to 0.5 , as was discussed in section 4 . Once $B$ and $w$ have been chosen, the $E^{\prime}$ and $\alpha^{\prime}$ values can be found using figure 4 or equivalently equation 15 . Finally, it is necessary to select a value for $Z_{M}$ by choosing a decay probability $\phi$, which determines the length of the HD simulation periods. Noting that $\phi / w$ is the expected fraction of bias values above $Z_{M}$ and we can suggest $\phi<w / 1000$ for a good sampling on each HD simulation period. Then using the selected $\phi$ and equation 20, the value for $Z_{M}$ is obtained.

Once the three parameters $B, w$ and $Z_{M}$ are defined, the first MD simulation begins, and the criterion for the attainment of the pseudo equilibrium condition is checked every $l$ steps. When the PEC condition is satisfied, the values for $\bar{X}$ and $\sigma(X)$ are evaluated and the parameters $\alpha$ and $E$ and are computed through equations 17 and 16 respectively. 


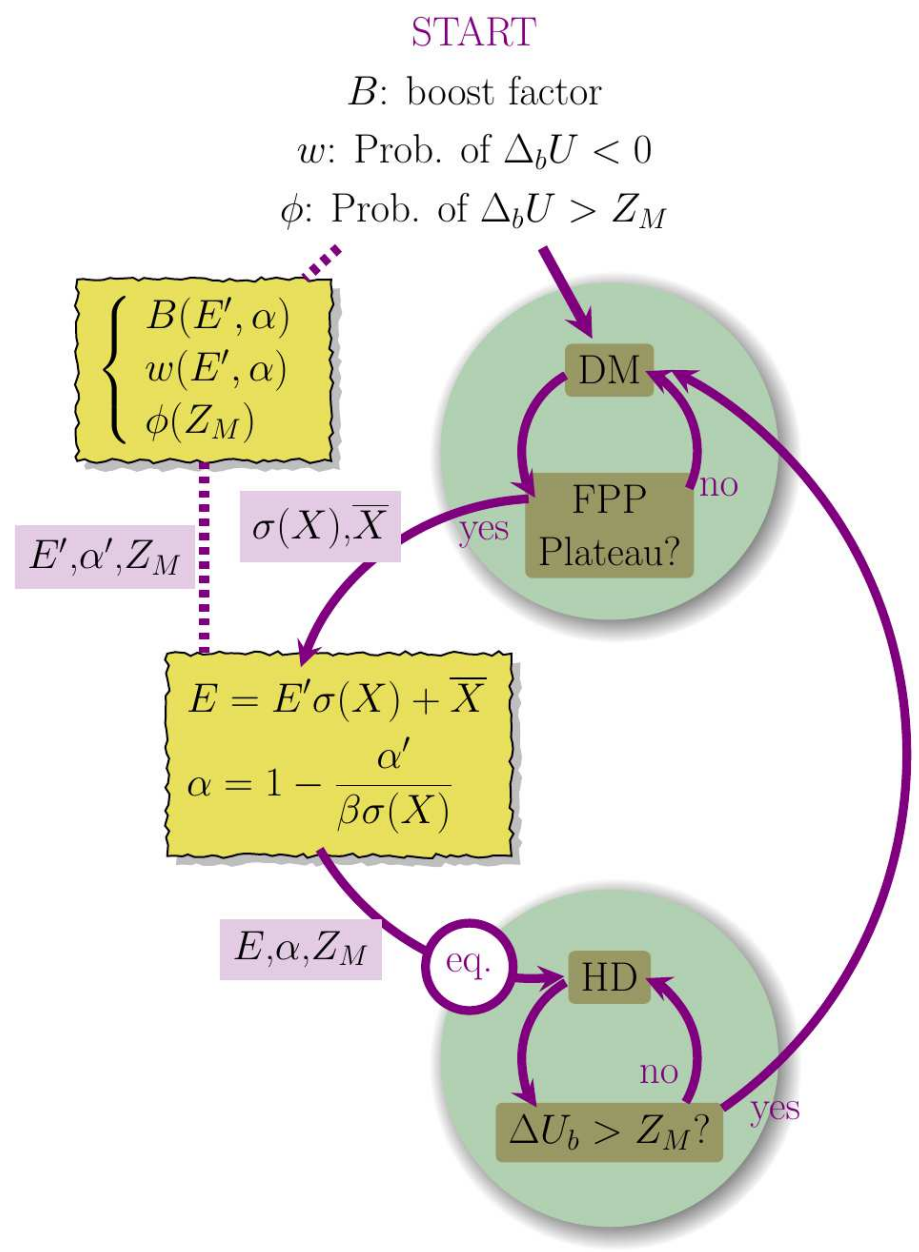

Figure 5: Flow chart of the hybrid HD-MD method. The main input parameter are the desired boost factor $B$, the desired non-zero bias probability $w$ and the decay probability $\phi$ to stop the HD simulation. 
Then, the HD simulation starts. Since the first $n$ steps of HD do not belong to an ergodic sampling of the biased PES, but rather to the unbiased one, these steps are considered an equilibration stage and are discarded. Note that suitable values for $l$ and $n$ can be estimated from a straightforward study of the energy autocorrelation function, a common practice in most MD studies. Finally, the HD simulation proceeds until the condition $\Delta U_{b}>Z_{M}$ is achieved and the MD starts again.

\section{Testing the method for a two-dimensional model po- tential}

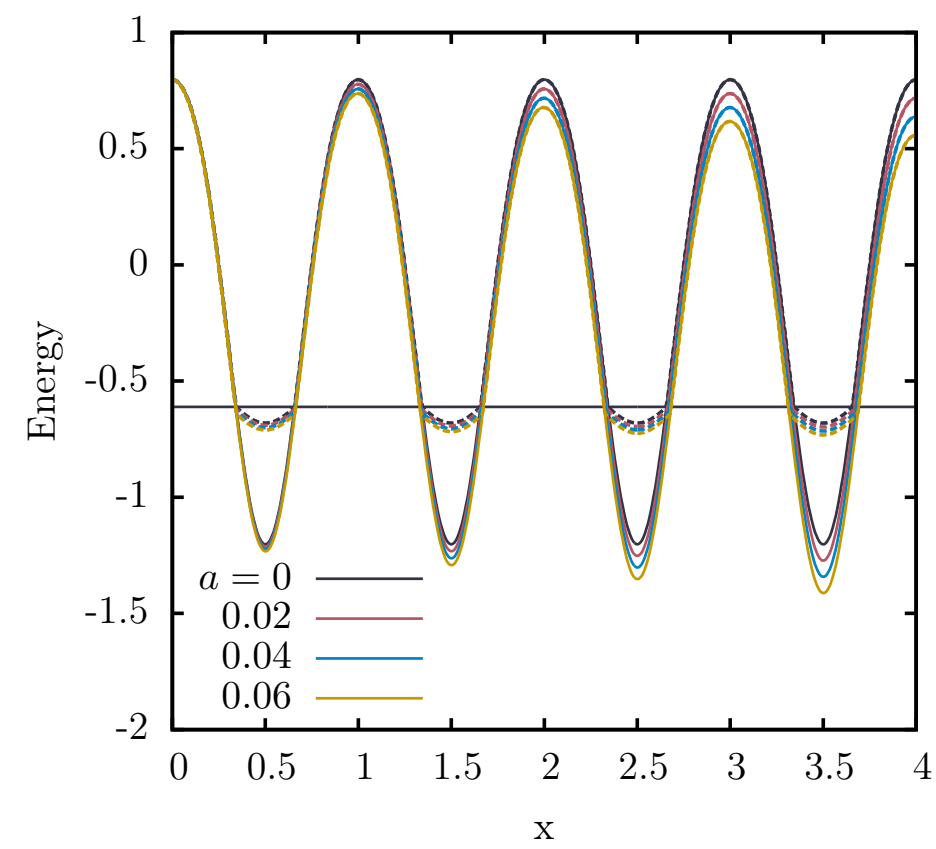

Figure 6: Minimum-energy path along the $x$-direction of the two-dimensional model potential defined in equation 21. Dashed lines correspond to the biased PES obtained using the bias function 6 with $E=-0.61$ and $\alpha=0.12$.

In order to illustrate some of the capabilities of the hybrid HD-MD method we apply it 
to study the motion of a particle in the two-dimensional model potential given by:

$$
U(x, y)=\cos (2 \pi x)(1+4 y)+2 \pi^{2} y^{2}-a x
$$

Excluding the last term, this is the potential named as "type I" by Voter in his original publication of HD. ${ }^{11}$ The new term $-a x$ is intended to be a small perturbation that generates a tilt in the potential along the $x$ direction (see figure 8). Therefore, when $a \neq 0$ this term introduces a constant drift in the motion of the particle. We use 500 trajectories from pure MD simulations in order to measure the drift and diffusion coefficient along the $x$ direction for different values of $a$. In order to allow the particle to overcome the potential barriers we choose $k_{B} T=0.2$, and following reference 11 we fix a time step of 0.01 and a friction coefficient of 0.5 for the Langevin dynamics. For each trajectory, the total simulation time was $10^{6}$. The drift $\mu$ and diffusion coefficient D were computed trough the respective equations:

$$
\begin{aligned}
\mu & \equiv \frac{d}{d t}\langle x(t)-x(0)\rangle \\
D & \equiv \frac{1}{2} \frac{d}{d t}\left\langle(x(t)-x(0)-\mu t)^{2}\right\rangle
\end{aligned}
$$

The results are reported in the columns headed with the label "MD" in table 1. It should be noticed that for $a=0$ the diffusion coefficient obtained is in good agreement with the value of $(4.5 \pm 0.4) 10^{-5}$ reported previously by Voter. ${ }^{11}$

Table 1 also reports the drift and diffusion coefficient computed from 500 trajectories simulated with HD using the bias function of eq 6 with $E=-0.61$ and $\alpha=0.12$. It can be noticed that for $a=0$ there is a good agreement between the values obtained from MD and HD. The difference between these values can be taken as a consequence of the TST assumption on the HD framework, as was demonstrated in reference 11. On the other hand, when $a \neq 0$ the direct implementation of HD lead to an unbounded increase of the boost factor in the trajectories, ending in the poor sampling of the basins, the increment of the 
number of multiple crossing events and the persistent violation of the zero bias condition at the transition states. This can be clearly noticed in the case of $a=0.1$ where the simulation time per trajectory (fixed to $10^{6}$ ) is enough to achieve the described regimen. Furthermore, it is even not possible to fit a constant value for $\mu$ and $D$ to the result of this simulations, as it can be seen from the non-linear dependence of the mean displacement in figure 7 .

Finally, we computed the drift and diffusion coefficient from 500 trajectories through the hybrid HD-MD method. The parameters for these simulations were $B=4, w=0.9$ and $\phi=10^{-3}$. The results are also shown in table 1 , where it can be observed a good agreement with the MD even when $a$ is bigger. This agreement arise due to the capability of the method to recalibrate $E$ and $\alpha$ ensuring a constant boost factor along the trajectory. As was mentioned above, the differences with MD could be explained from the basis of the HD in TST. It is expected that this difference increases with $a$ the probability to observe multiple crossing events also increases. Nevertheless, a linear dependence of the mean displacement with time is obtained in all the studied cases as can be see in figure 7 for $a=0.1$.

Table 1: Comparison between the drift $\mu$ and diffusion coefficient $D$ obtained by MD, HD and hybrid HD-MD. The simulation time was fixed to $10^{6}$ per trajectory. The dashes in the last row indicate that it was not possible to assign a constant value.

\begin{tabular}{|c|c|c|c|c|c|c|}
\cline { 2 - 7 } \multicolumn{1}{c|}{} & \multicolumn{3}{c|}{$\mu\left(10^{-5}\right)$} & \multicolumn{3}{c|}{$D\left(10^{-5}\right)$} \\
\hline$a$ & MD & HDMD & HD & MD & HDMD & HD \\
\hline 0 & -0.02 & 0.08 & 0.05 & 4.43 & 4.82 & 5.03 \\
0.02 & 0.44 & 0.49 & 0.61 & 4.41 & 4.98 & 4.89 \\
0.04 & 0.89 & 0.83 & 1.07 & 4.31 & 5.20 & 5.10 \\
0.06 & 1.34 & 1.38 & 1.56 & 4.47 & 5.44 & 5.20 \\
0.1 & 2.30 & 2.46 & - & 4.53 & 6.16 & - \\
\hline
\end{tabular}




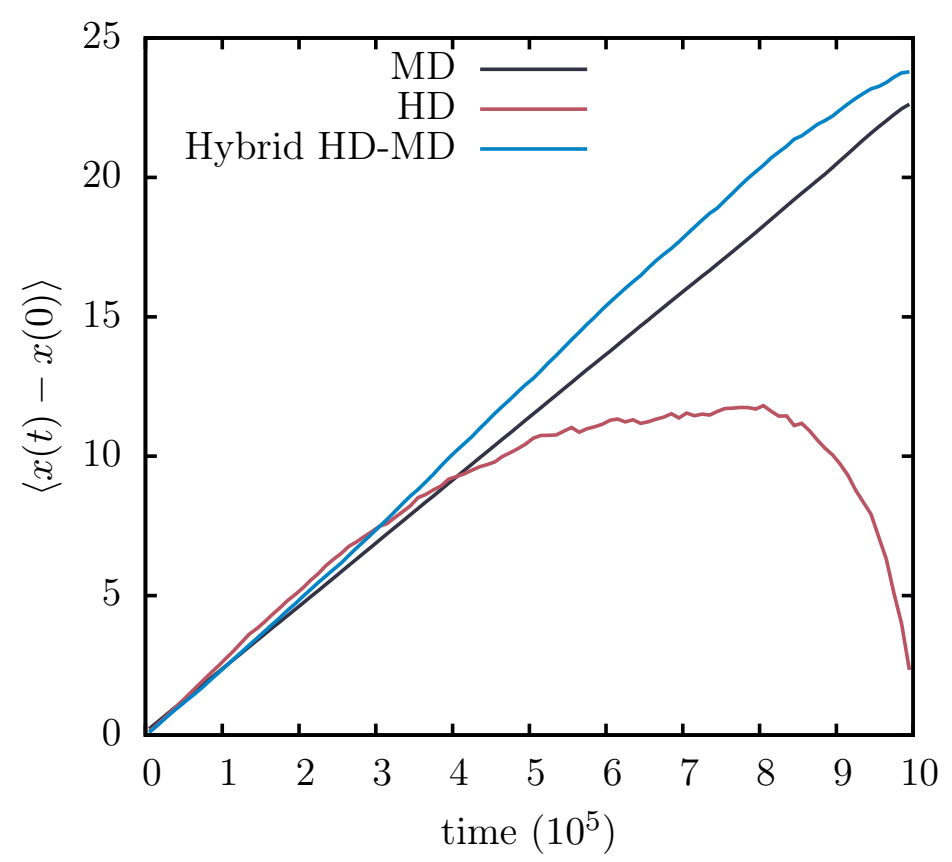

Figure 7: Mean displacement along the $\mathrm{x}$ direction for the model potential of equation 21 computed using 500 trajectories from pure MD, a direct application of HD and the hybrid HD-MD method.

\section{Testing the method for the coalescence of nanopar- ticles}

We present here the aplication of the hybrid HD-MD method to study the coalescence process of two metal nanoparticles (NPs) of $42 \mathrm{Au}$ atoms and 13 Co atoms respectively. The problem of NPs coalescence is a hot research area ${ }^{36-44}$ as well as the study of bimetalic NPs and their remarkable features. ${ }^{45-50}$ For the coalescence process presented here, the simulation results can be directly compared with those obtained from straightforward MD simulations performed in our previous work. ${ }^{31}$

Figure 8a presents an example of the energy profile for a hybrid HD-MD trajectory for the same initial conditions as those of the results presented in figure 1. The parameters of this simulation were $B=10, w=0.5$ and $\phi=410^{-6}$. Note that until completing the first $5 \mathrm{~ns}$, the energy traces of figures 1 and 8 are the same. After this time the HD begins, in synchrony with the plateau found in the FPP (see figure $2 \mathrm{~b}$ ). The yellow traces correspond 


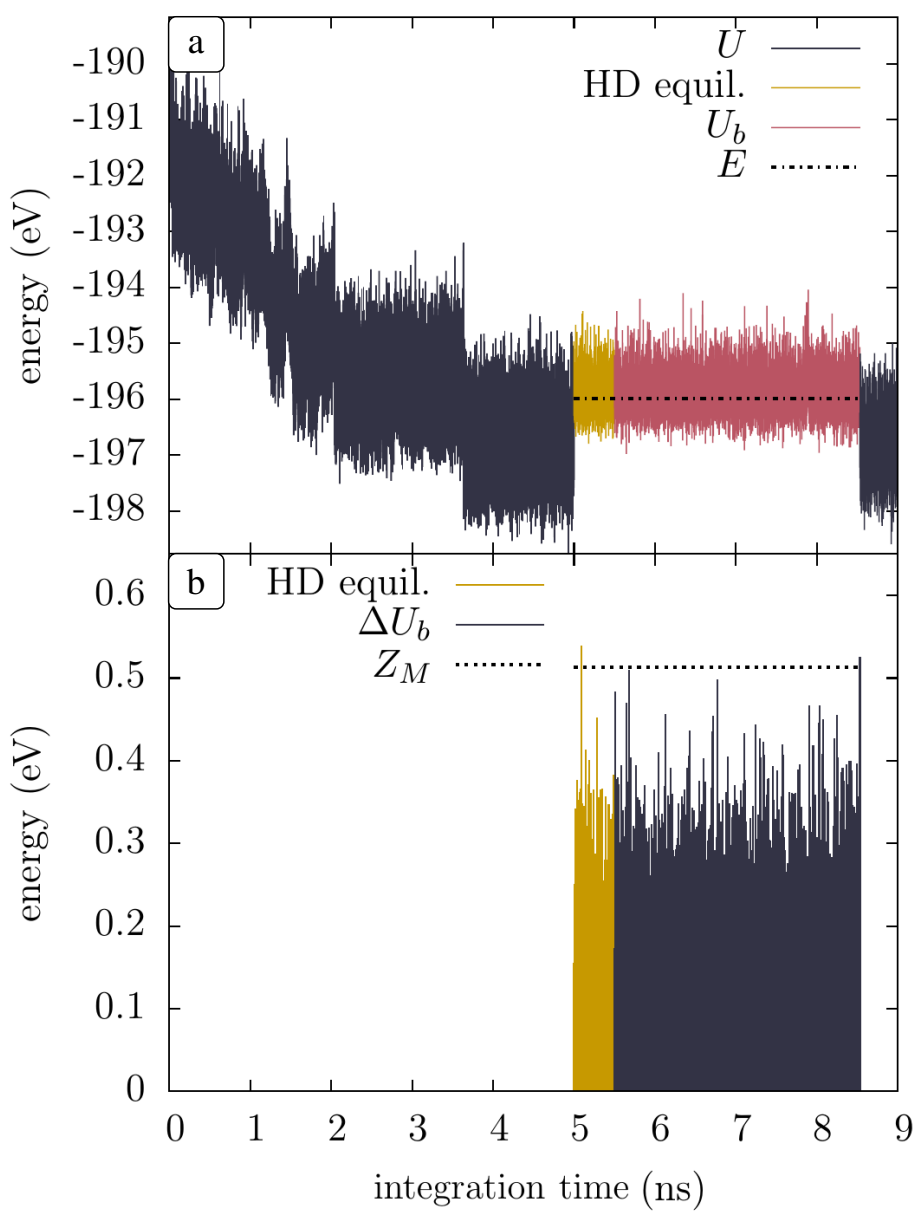

Figure 8: a): Energy trace for the same phenomenon of coalescence as that analyzed in figure 1, but resulting from the hybrid HD-MD method. b): The corresponding values of the bias. The time axis correspond to the "integration time" (without taking into account the boost). 
to the equilibration steps and the black dashed line denotes the on-the-fly computed value for $E$. Note that $E$ is located close to the center of the energy trace, as expected for the input $w=0.5$. It is important to note that the $\mathrm{x}$-axis corresponds to the "integration time" $t$ (without boost) and not to the "boosted time". In the later case the energy values would appear spaced with irregular, longer time steps. Figure $8 \mathrm{~b}$ shows the profile of the bias observed and the horizontal dashed line indicate the $Z_{M}$ value computed on-the-fly. When the bias overcomes this threshold $(t \approx 8.5 \mathrm{~ns})$ the HD is stopped, and the MD start again. It can be noticed in this figure how during the equilibration steps, $Z_{M}$ is overcome $(t \approx 5.1 \mathrm{~ns})$ without leading to the switch off of the HD.

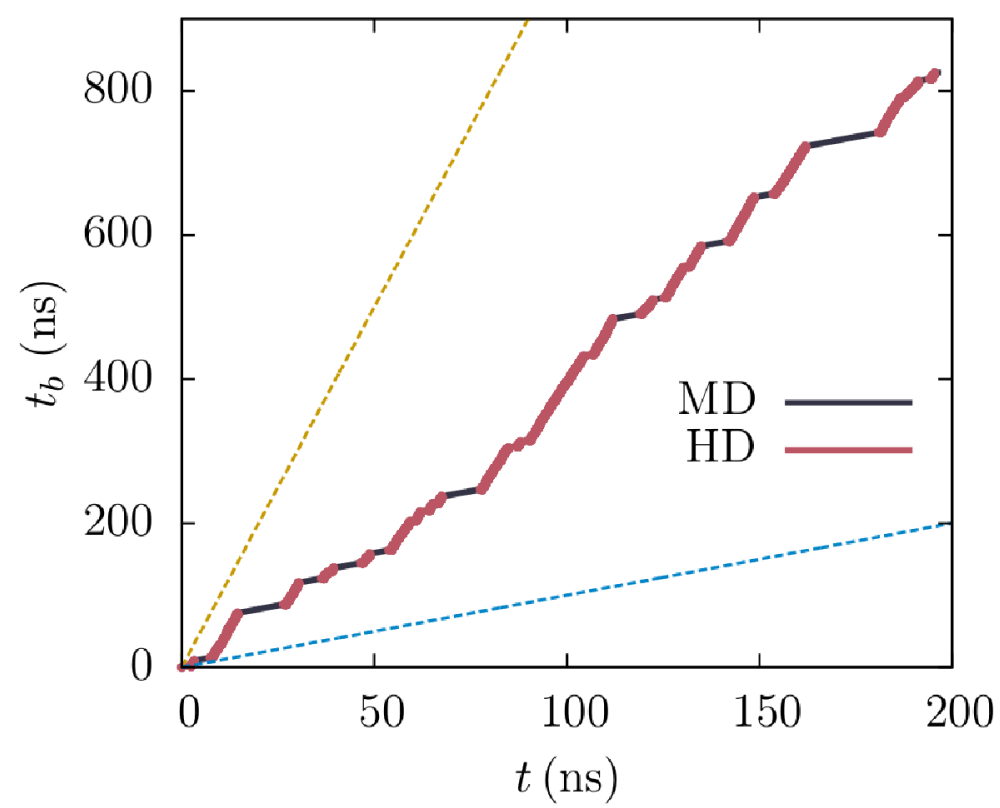

Figure 9: Boosted time $t_{b}$ vs integration time $t$ for the hybrid HD-MD simulation of the coalescence process, using $B=10$. Dashed lines show the expected slope for a pure MD simulation (blue) and for a pure (hypotetic) HD with a boost factor of 10 (orange).

It should be pointed out an important difference between the global boost factor obtained by the HD-MD hybrid method and the boost factor obtained in each HD stage. We will call $t^{H D}$ to the integration time spent only in the HD periods and $t_{b}^{H D}$ to the corresponding boosted time. On the other hand we will call $t$ to the total integration time of the hybrid HD-MD simulation and $t_{b}$ to the corresponding boosted time. Therefore, it is expected that 
$B \approx t_{b}^{H D} / t^{H D}$ and $t_{b} / t \leq B$ because of the simulation time spent in regular MD. Figure 9 shows a plot of $t_{b}$ vs $t$, where it is possible to differentiate the different periods of HD and MD simulations. It can be observed how the global slope of the simulation is higher than the slope of each MD stage, but is lower than the slope of each HD period which present the desired $B=10$.

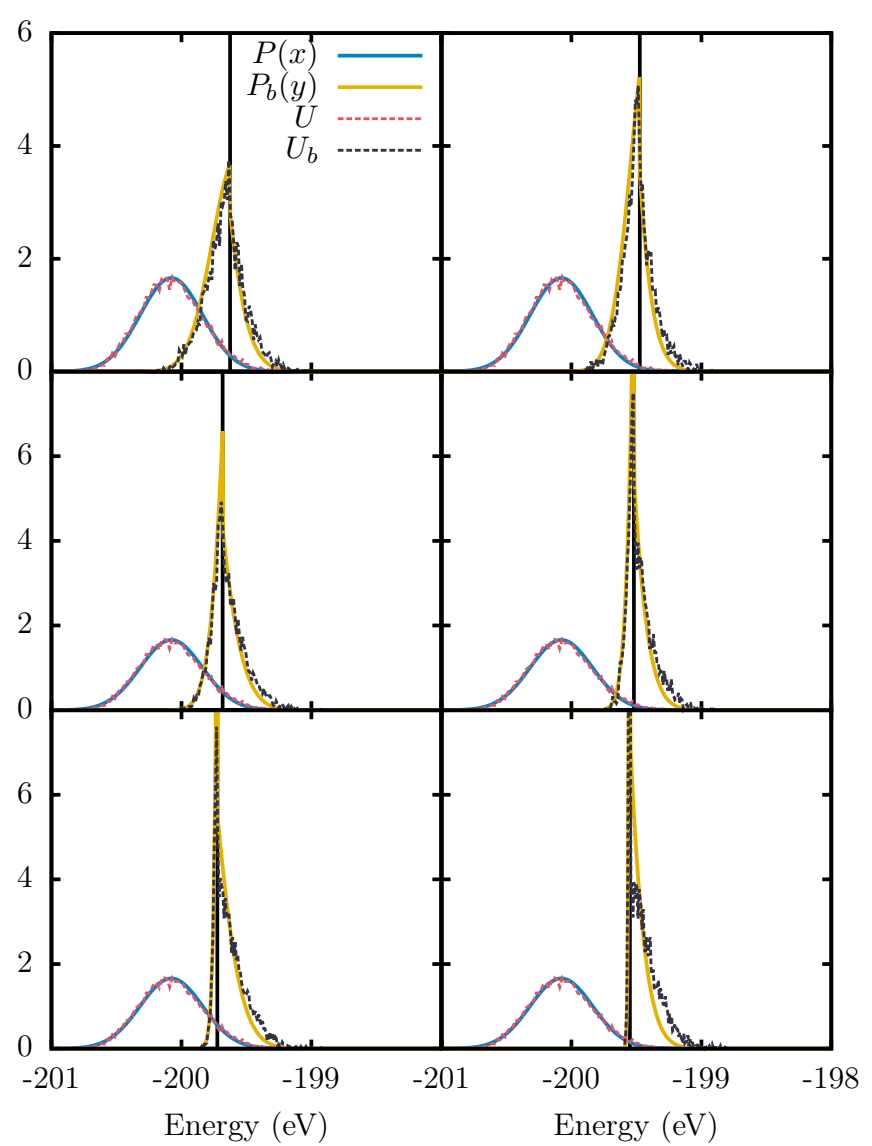

Figure 10: Energy distribution $P(x)$ and $P_{b}(x)$ for some $w$ and $B$ as is indicated in each frame. Continuous lines correspond to the gaussian fit (blue) and the prediction according to equation 10 (yellow). The dashed lines correspond to the histograms measured during the MD (black) and the subsequent HD (red) simulations.

Figure 9 suggests a way to measure the power of a hybrid HD-MD simulation. It is possible to compare the input $B$ value with the measured ratio $t_{b}^{H D} / t^{H D}$. Simultaneously, it is also possible to compare the input $w$ value with the fraction of HD steps with a positive value of the bias. We performed this comparison for a certain set of $B$ and $w$ parameters, as 
presented in table 2. Better agreements are found for $w=0.5$, which is consistent with the discussions given above. On the other hand, there are larger deviations of the ratio $t_{b}^{H D} / t^{H D}$ from the preset value of $B$ for simulations with $w=0.3$ and $B>10$, as highlighted in red. This deviations are indicating a too aggressive acceleration which could lead to several issues, as for example, a bad statistics over $t_{b}^{H D}$ (see reference 11).

Table 2: Comparison between $B$ and $w$ input parameters and the measured equivalent quantities from the performed hybrid simulations. The slope $t_{b}^{H D} / t^{H D}$ is obtained through a linear fit of the $t_{b}^{H D}$ vs $t^{H D}$ plot.

\begin{tabular}{|c|c|c|c|c|c|c|c|}
\hline & \multicolumn{6}{|c|}{$B$} \\
\hline & & 10 & 50 & 100 & 10 & 50 & 100 \\
\hline \multirow{3}{*}{$w$} & 0.3 & 0.26 & 0.24 & 0.23 & 12.4 & 1318 & 323.0 \\
\hline & 0.5 & 0.45 & 0.40 & 0.40 & 10.3 & 55.7 & 111.6 \\
\hline & 0.7 & 0.64 & 0.60 & 0.59 & 8.79 & 32.6 & 55.1 \\
\hline & & \multicolumn{3}{|c|}{$\operatorname{Prob}\left(\Delta U_{b}>0\right)$} & \multicolumn{3}{|c|}{$t_{b}^{H D} / t^{H D}$} \\
\hline
\end{tabular}

In addition to the simple test of table 2, a full comparison between predicted and obtained energy distributions $P(x), P_{b}(y)$ and $P_{\Delta}(z)$ was performed. The histograms of potential energy for a HD simulation period and the corresponding histogram for the immediately preceding MD simulation are plotted in figure 10, for differents $w$ and $B$ input parameters. The respective approximated $P(x)$ and predicted $P_{b}(y)$ densities are also shown in the plot. In all cases, the gaussian shaped $P(x)$ fits very well the main part of the MD energy distribution. In the case of $P_{b}(y)$, a better agreement is found for energies below $E$. This can be understood considering that this portion of $P_{b}(y)$ is predicted using the gaussian approximation of $P(x)$ (equation 14 ) at energies closer to $\langle X\rangle$, where the approximation is better. ${ }^{35}$ Far away from the center of $P(x)$, as it is the case of energies above $E$, the gaussian approximation may not be good, and therefore the predicted $P_{b}(y)$ for these energies is expected to show some deviations, as can be seen in the figure. When $w$ is small and/or $B$ is big, the computed $E$ could be many $\sigma(X)$ times far from $\bar{X}$, and the discrepancy of $P_{b}(y)$ above $E$ is expected to be bigger. For the same reason, similar discrepancies can be observed on the predicted distribution of the bias $P_{\Delta}(z)$ near cero (see supplementary information), the region of the 
distribution related to $P(x)$ with energies close to $E$. Despite this details, figure 10 shows that a good overall prediction of $P_{b}(y)$ may be obtained if suitable $w$ and $B$ parameters are selected carefully.

The time behavior of a NP coalescence process was studied by straightforward MD in our previous work. ${ }^{31}$ In that work, a histogram of waiting times for the formation of the final core-shell structure was constructed using $256 \mathrm{MD}$ trajectories. A replica of this histogram is shown in figure 11a. Two peaks (labeled as I and II) can be seen in this figure. In the aforementioned work, we found that peak II arises from some particular set of trapped states that delay the process of core-shell formation. In order to study the predictive power of the present method, we constructed the same histogram by means of hybrid HD-MD simulations. Therefore, with $w=0.5, B=50$ and $\phi=710^{-6}$ as input parameters, 256 hybrid HD-MD simulations where performed with different random seeds and orientations of the initial NP, as done in the reference work. Two kind of histograms may be constructed with the results of the present simulations: the histogram using the integration time $t$ and the histogram using the boosted time $t_{b}$. These two histograms are shown in figure $11 \mathrm{~b}$ and $11 \mathrm{c}$ respectively. In the former, only a broad peak around $30 \mathrm{~ns}$ is found and there is no evidence for peak II. This means that in the hybrid simulations the trajectories can quickly escape from the trapped states associated with this peak. Moreover, it can be observed in figure 11(d) that peak II reappears, so that the presence of the trapped system is reflected after taking in account the boosted time. The occurrence of this peak is an exciting result, since it is an indication that accelerated dynamics may recover features of a very complex process, involving a large number of degrees of freedom (165).

\section{Conclusions}

We propose an on-the-fly algorithm to switch between ordinary and accelerated molecular dynamics during the course of a simulation. This automatic interchange requires a minimal 


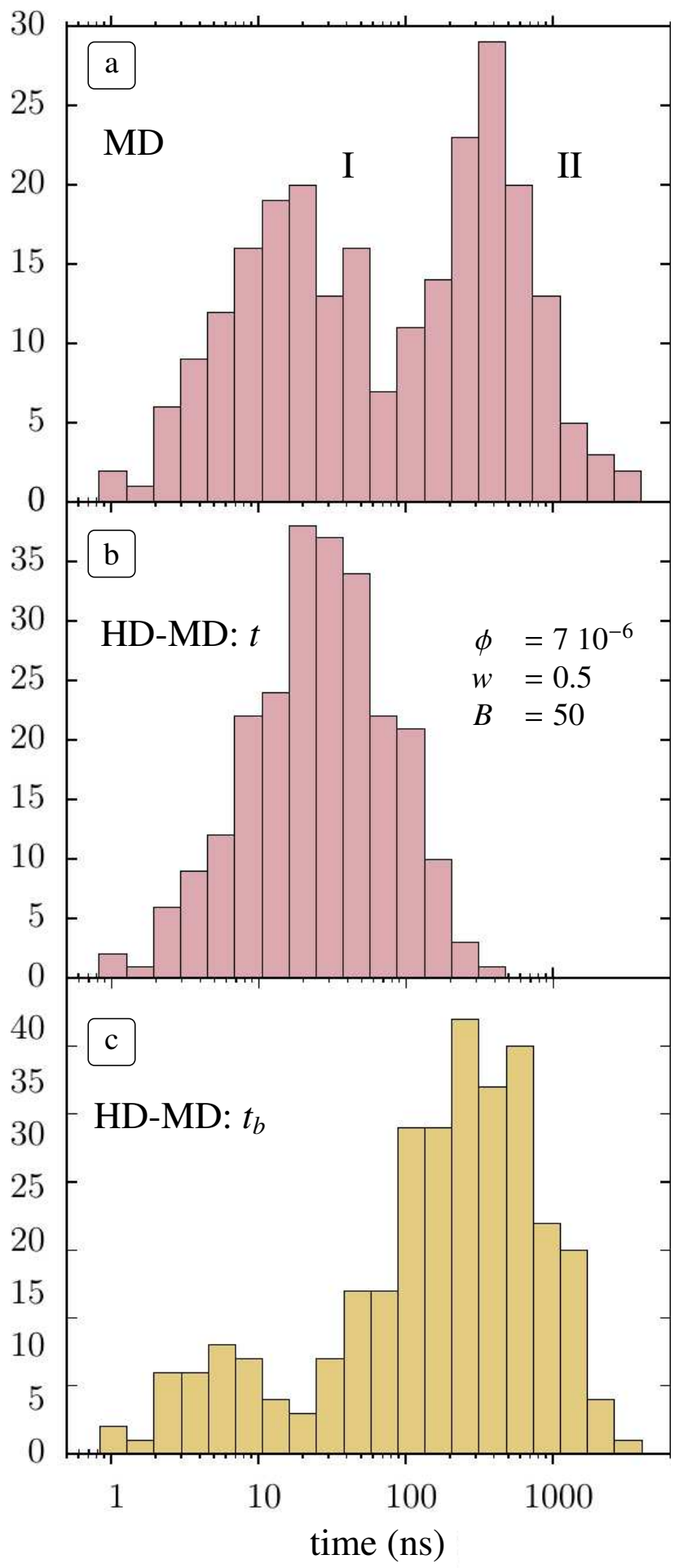

Figure 11: (a): Distribution of waiting times for the formation of the final core-shell structure built from pure MD, taken as reference from our recent work. ${ }^{31}$ (b) and (c): Same as (a) but using "integration" and "boosted" times respectively obtained from the hybrid HD-MD simulations. 
computational cost and allows to adapt the bias parameters to the different trapped states visited by the system. Furthermore, this method prevents the application of the hyperdynamics scheme in evolution periods that do not fullfill the pseudo equilibrium condition required for the validity of the transition state theory. An analytical treatment of the different criteria imposed to regulate the algorithm was achieved by using a simple bias function, approximating the unbiased energy distribution by a gaussian function. Even with this approximation, the method successfully predicts the long time characteristics of the coalescence process of two metallic nanoparticles (165 degrees of freedoms). As far as we know, this is the first time that an hyperdynamics scheme using a bias potential of the type given in equations 5-6 can recover relevant time information for such a complex process.

The present results on nanoparticle coalescence heuristically show that it is possible to obtain sets of trajectories where the kinetics of particle coalescence is mostly preserved, even for this complex system. On the other hand, many hyperdynamic strategies that formally guarantee the condition of bias nullity at the transition state are not computationally efficient to take advantage of the acceleration obtained. Thus, although the present methodology is clearly not the optimal one concerning hyperdynamics, it opens the possibility to observe many relevant trajectories using a computationally efficient method, without the need to define a particular set of collective variables. Further research is desirable to analyze the applicability of the present methodology to other complex systems. 


\section{Appendix: Prediction of the HD energy distribu- tion}

The density of states of the unbiased and biased systems are respectively given by:

$$
\begin{aligned}
\omega(x) & \equiv \int_{\Omega} \delta(x-U(\mathbf{r})) d \mathbf{r} \\
\omega_{b}(y) & \equiv \int_{\Omega} \delta\left(y-U_{b}(\mathbf{r})\right) d \mathbf{r}
\end{aligned}
$$

Where $\Omega$ denotes the configuration space. If the bias function used is a energy-based bias function (equation 4), it is possible to write:

$$
U_{b}(\mathbf{r})=U(\mathbf{r})+g(U(\mathbf{r})) \equiv f(U(\mathbf{r}))
$$

Then, the number of states with energy $y=U_{b}(\mathbf{r})$ can be also recovered by taking into account all the energies $x=U(\mathbf{r})$ that satisfy 26. In other words:

$$
\begin{aligned}
\int_{-\infty}^{\infty} \omega(x) \delta & (y-f(x)) d x \\
& =\int_{-\infty}^{\infty} \int_{\Omega} \delta(x-U(\mathbf{r})) \delta(y-f(x)) d \mathbf{r} d x \\
& =\int_{\Omega} \delta(y-f(U(\mathbf{r}))) d \mathbf{r} \\
& =\omega_{b}(y)
\end{aligned}
$$

This equation sets the relationship between the density of states of the biased and unbiased system and can be used together with equation 8 and 9 to get:

$$
P_{b}(y)=\frac{Q}{Q_{b}} \int_{\infty}^{\infty} P(x) e^{-\beta(y-x)} \delta(y-f(x)) d x
$$

which relates the probability distribution of variables $X$ and $Y$. If the function $f(x)$ is 
invertible, an especial case of equation 31 arises by changing the variable of integration trough $x=f^{-1}\left(y^{\prime}\right)$ :

$$
P_{b}(y)=\frac{Q}{Q_{b}} e^{-\beta\left(y-f^{-1}(y)\right)}\left|\frac{d f^{-1}(y)}{d y}\right| P\left(f^{-1}(y)\right)
$$

This is the case of the bias function presented in equation 6 , used in this work. The use of this bias function in the above equation leads to equation 10.

Equation 32, or even the more general case of equation 31, gives the possibility to predict any property of the biased system that depends on its energy distribution. This could be used to improve the simulation parameters of the HD simulation before it starts. As it is shown in the present work, some conditions to achieve a well behaved acceleration or even the desired boost factor can be fixed in this way.

Let us define $Z$ as the random variable associated with the bias values observed during the HD simulation. The $Z$ values arise by sampling $Y$ through the function:

$$
Z \equiv Y-f^{-1}(Y) \equiv h(Y)
$$

It is possible to use this function with the random variable transformation theorem ${ }^{51}$ to obtain the distribution function $P_{\Delta}(z)$ of $Z$ from $P_{b}(y)$ :

$$
P_{\Delta}(z)=\int_{-\infty}^{\infty} P_{b}(y) \delta(z-h(y)) d y
$$

Then, $P_{\Delta}(z)$ can be predicted by computing $P_{b}(y)$ from the MD simulation via equation 31 or, in the case of the bias function used in this work, replaced from equation 10 to obtain equation 18.

\section{Acknowledgement}

We acknowledge financial support from CONICET PIP: 112-200801-000983, Secyt Univer- 
sidad Nacional de Córdoba, Program BID (PICT-BICENTENARIO-2010-123), and PME: 2006-01581.

\section{Supporting Information Available}

Predicted and obtained $P_{\Delta}(z)$ distribution of the magnitude of the bias for some $w$ and $B$ parameters. This material is available free of charge via the Internet at http://pubs.acs . org/.

\section{References}

(1) Chipot, C., Pohorille, A., Eds. Free energy calculations. Theory and applications in chemistry and biology; Springer: Berlin, 2007.

(2) Chipot, C. WIREs Comput. Mol. Sci. 2014, 4, 71.

(3) Hansen, N.; van Gunsteren, W. F. J. Chem. Theory Comput. 2014, 10, 2632.

(4) Wales, D. J.; Bogdan, T. V. J. Phys. Chem. B 2006, 110, 20765.

(5) Abrams, C. F.; Vanden-Eijnden, E. Chem. Phys. Lett. 2012, 547, 114.

(6) Maragliano, L.; Vanden-Eijnden, E. J. Chem. Phys. 2008, 128, 184110.

(7) Maragliano, L.; Fischer, A.; Vanden-Eijnden, E.; Ciccotti, G. J. Chem. Phys. 2006, $125,24106$.

(8) Maragliano, L.; Vanden-Eijnden, E. Chem. Phys. Lett. 2006, 426, 168.

(9) Laio, A.; Gervasio, F. L. Reports Prog. Phys. 2008, 71, 126601.

(10) Darve, E.; Rodríguez-Gómez, D.; Pohorille, A. J. Chem. Phys. 2008, 128, 144120.

(11) Voter, A. F. J. Chem. Phys. 1996, 106, 4665. 
(12) Voter, A. F. Phys. Rev. Lett. 1997, 78, 3908.

(13) Torrie, G.; Valleau, J. J. Comput. Phys. 1977, 23, 187.

(14) Kim, S. Y.; Perez, D.; Voter, A. F. J. Chem. Phys. 2013, 139, 144110.

(15) Tiwary, P.; van de Walle, A. Phys. Rev. B 2011, 84.

(16) Xin, Y.; Doshi, U.; Hamelberg, D. J. Chem. Phys. 2010, 132, 224101.

(17) Kim, W. K.; Falk, M. L. Model. Simul. Mater. Sci. Eng. 2010, 18, 034003.

(18) Fichthorn, K. A.; Miron, R. A.; Wang, Y.; Tiwary, Y. J. Phys. Condens. Matter 2009, 21, 084212 .

(19) Perez, D.; Voter, A. F. Accelerating atomistic simulations through self-learning bond-boost hyperdynamics.; 2008. http://permalink.lanl.gov/object/tr?what=info:lanlrepo/lareport/LA-UR-08-05519.

(20) de Oliveira, C. A. F.; Hamelberg, D.; McCammon, J. A. J. Chem. Theory Comput. 2008, 4, 1516 .

(21) Becker, K. E.; Fichthorn, K. A. J. Chem. Phys. 2006, 125, 184706.

(22) Hamelberg, D.; Shen, T.; Andrew McCammon, J. J. Chem. Phys. 2005, 122, 241103.

(23) Hamelberg, D.; Mongan, J.; McCammon, J. A. J. Chem. Phys. 2004, 120, 11919.

(24) Miron, R.; Fichthorn, K. Phys. Rev. Lett. 2004, 93, 128301.

(25) Miron, R. A.; Fichthorn, K. A. J. Chem. Phys. 2003, 119, 6210.

(26) Sminchisescu, C.; Triggs, B. Comput. VisionECCV 2002; Springer: Berlin, 2002; Vol. $2350 ;$ p 769.

(27) Wang, J.-C.; Pal, S.; Fichthorn, K. A. Phys. Rev. B 2001, 63, 085403. 
(28) Gong, X.; Wilkins, J. Phys. Rev. B 1999, 59, 54.

(29) Pal, S.; Fichthorn, K. A. Chem. Eng. J. 1999, 74, 77.

(30) Steiner, M.; Genilloud, P.-A.; Wilkins, J. Phys. Rev. B 1998, 57, 10236.

(31) Paz, S. A.; Leiva, E. P. Chem. Phys. Lett. 2014, 595-596, 87.

(32) Flyvbjerg, H.; Petersen, H. G. J. Chem. Phys. 1989, 91, 461.

(33) Chen, F.; Curley, B. C.; Rossi, G.; Johnston, R. L. J. Phys. Chem. C 2007, 111, 9157.

(34) Kent, I.; David, R.; Muller, R.; Anderson, A.; Goddard III, W.; Feldmann, M. J. Comput. Chem. 2007, 28, 2309.

(35) Reif, F. Fundamentals of statistical and thermal physics; McGraw-Hill: Tokyo, 1965.

(36) Mariscal, M. M.; Mayoral, A.; Olmos-Asar, J. A.; Magen, C.; Mejía-Rosales, S.; PérezTijerina, E.; Yacamán, M. J. Nanoscale 2011, 3, 5013.

(37) Zheng, H.; Smith, R. K.; Jun, Y.-W.; Kisielowski, C.; Dahmen, U.; Alivisatos, a. P. Science 2009, 324, 1309.

(38) Liu, H.; Yacamán, M. J.; Perez, R.; Ascencio, J. A. Appl. Phys. A 2003, 77, 63.

(39) Yacamán, M. J.; Gutierrez-Wing, C.; Miki, M.; Yang, D.-Q.; Piyakis, K. N.; Sacher, E. J. Phys. Chem. B 2005, 109, 9703.

(40) Paz, S. A.; Leiva, E. P. M.; Jellinek, J.; Mariscal, M. M. J. Chem. Phys. 2011, 134, 094701.

(41) Mariscal, M. M.; Oldani, N. a.; Dassie, S. A.; Leiva, E. P. M. Faraday Discuss. 2008, $138,89$.

(42) Ferrando, R.; Jellinek, J.; Johnston, R. L. Chem. Rev. 2008, 108, 845. 
(43) Mariscal, M. M.; Dassie, S. A.; Leiva, E. P. M. J. Chem. Phys. 2005, 123, 184505.

(44) Baletto, F.; Ferrando, R. Rev. Mod. Phys. 2005, 77, 371.

(45) Khanal, S.; Spitale, A.; Bhattarai, N.; Bahena, D.; Velazquez-Salazar, J. J.; MejíaRosales, S.; M Mariscal, M.; José-Yacaman, M. Beilstein J. Nanotechnol. 2014, 5, 1371.

(46) Bochicchio, D.; Ferrando, R. Phys. Rev. B 2013, 87, 165435.

(47) Rapallo, A.; Olmos-Asar, J. A.; Oviedo, O. A.; Ludueña, M.; Ferrando, R.; Mariscal, M. M. J. Phys. Chem. C 2012, 116, 17210.

(48) Parsina, I.; DiPaola, C.; Baletto, F. Nanoscale 2012, 4, 1160.

(49) Parsina, I.; Baletto, F. J. Phys. Chem. C 2010, 114, 1504.

(50) Langlois, C.; Li, Z. L.; Yuan, J.; Alloyeau, D.; Nelayah, J.; Bochicchio, D.; Ferrando, R.; Ricolleau, C. Nanoscale 2012, 4, 3381.

(51) Gillespie, D. Am. J. Phys. 1983, 51, 520. 


\section{Graphical TOC Entry}

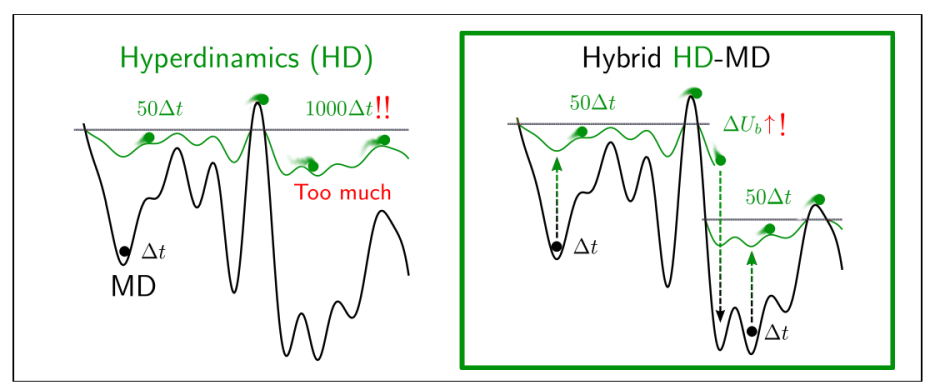

